\title{
MULTIDIMENSIONAL EXTENSION OF MMSE LINEAR ADAPTIVE RECEIVER FOR DSSS SYSTEMS
}

\author{
Julio E. Castro
}

James P. LeBlanc

\author{
Klipsch School of ECE \\ New Mexico State University \\ Las Cruces, NM 88001 \\ julcastr@nmsu.edu_leblanc@nmsu.edu
}

Predrag Rapajic

\author{
Telecommunications Eng., RSISE \\ The Australian National University \\ Canberra, Australia \\ prapajic@syseng.anu.edu.au
}

\begin{abstract}
In this paper we extend the single channel adaptive linear receiver (ALR) to the multidimensional case. The extension is used for the cancellation of strong spatially-distributed narrowband interference in direct sequence spread spectrum communications. Simulations show a gain of $8 \mathrm{~dB}$ for the case of two interferences occupying $30 \%$ of the bandwidth of the spread spectrum signal.
\end{abstract}

\section{INTRODUCTION}

The use of antenna arrays for digital mobile communications has received increasing attention in the communication research community in recent years. In particular, several researchers have concentrated on the use of antenna arrays for the reception of code division multiple access (CDMA) signals corrupted by multipath, additive white Gaussian noise (AWGN), multi access interference (MAI) and narrowband interference (NBI) [1] [2]. However, the research in this area has focused on the important aspect of using antenna arrays for the cancellation of MAI and acceptable power levels of NBI. We analyze the BER performance of an uniform antenna array fitted with adaptive linear filters for the excision of strong narrowband interference. The NBI is assumed to be time invariant and spatially distributed. Notice that the aggregate power contribution of spatially located interferences may occupy a large fraction of the signal bandwidth severely corrupting the signal of interest.

The proposed system consists of a $M$-element uniform antenna array, $M$ adaptive linear filters, and a decision device as shown in Figure 1. We present the multidimensional extension to the receiver first introduced in [3]. The NBI cancellation properties of the adaptive linear receiver (ALR) have been studied in [4]. We call this structure multidimensional adaptive linear receiver (MALR). Notice that MALR is structurally similar to the canonical broadband antenna array. However, for this system, a block of $L$ chip samples is processed at a time and the time span of the receiver is greater than or equal to a symbol period. Thus, the system generates an output every symbol period.

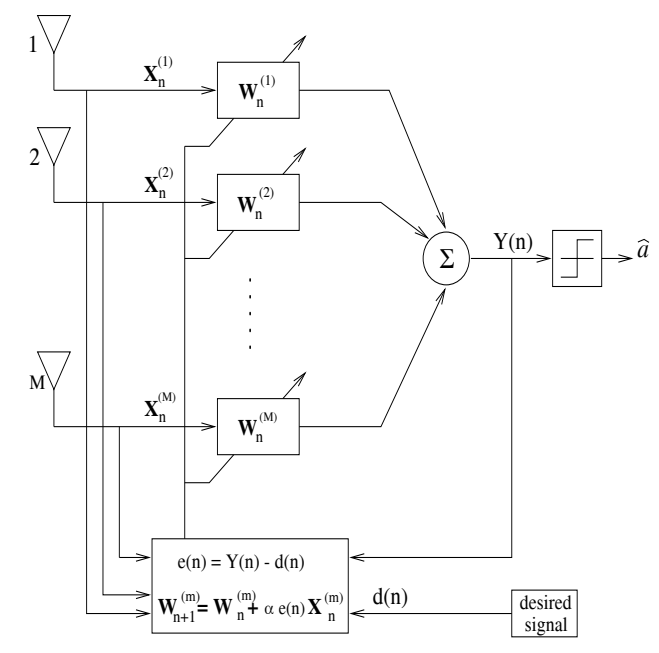

Figure 1: Multidimensional Adaptive Linear Receiver

\section{SIGNAL MODEL}

The assumed signaling method is direct sequence spread spectrum (DSSS). In general, the transmitted signal is corrupted by multipath propagation, AWGN $v(t)$ with variance $\sigma_{v}^{2}$, and NBI $\iota(t)$. In DSSS transmission, the $i$-th symbol, $a(i)$, is multiplied by the signature waveform,

$$
s(t)=\sum_{l=1}^{L} c(l) p\left(t-l T_{c}\right)
$$

where $L$ is the signature length, $T_{c}$ is the chip period, $c(l)$ is the $l$-th chip of the PN sequence, and $p(t)$ is the chip pulse. The symbols $a(i)$ represent zero mean, independent random variables. We also assume that the AWGN samples $v(n)$ 
and symbols $a(i)$ are independent. The transmitted signal is given by

$$
q(t)=a(i) \sum_{l=1}^{L} c(l) p\left(t-i L T_{c}-l T_{c}\right)
$$

In general, this waveform is passed through a multipath channel described by $h(t)=\sum_{p=1}^{P} g_{p}(t) \delta\left(t-\tau_{p}\right)$, where $P$ is the number of paths and $g_{p}(t)$ and $\tau_{p}$ are the complex attenuations and path delays, respectively. However, we assume that the channel does not change within a symbol period. The received signal at the $m$-th antenna sensor is given by $r_{m}(t)=q(t) e^{j \phi_{m}} * h(t)+\sum_{k=1}^{K_{\iota}} \iota_{k}(t) e^{j \psi_{k, m}}+v(t)$, where $m=0,1, \ldots, M-1, K_{\iota}$ is the number of spatially distributed interferences, $*$ denotes the convolution operation, and $\phi_{m}$ and $\psi_{k, m}$ are the phase shift that result from the sensor separation. We assume that the array elements are a half-wavelength apart. Then $\phi_{m}=m \pi \sin \theta_{s}$ and $\psi_{k, m}=m \pi \sin \theta_{i_{k}}$, where $\theta_{s}$ and $\theta_{i_{k}}$ are the direction of arrival (DOA) of the signal of interest and the $k$-th NBI.

Our interference model consists of individual interference modeled as the superposition of different sinusoids of equal amplitude, equally spaced in frequency, and of uniform distributed phases occupying a fraction $\gamma$ of the signal bandwidth. This interference model is used in [5] [6]. The power spectral density of two interferers is shown in Figure 2. Notice that the interference occupies $30 \%$ of the signal bandwidth and it is $10 \mathrm{~dB}$ above the signal level. Thus, the received signal at the $m$-th antenna element is given by

$$
\begin{aligned}
r_{m}(t)= & a(i) \sum_{l=1}^{L} c(l) \tilde{h}\left(t-l T_{c}-i T-\tau\right) e^{j \phi_{m}} \\
& +\sum_{k=1}^{K_{L}} \iota_{k}(t) e^{j \psi_{k, m}}+v(t) .
\end{aligned}
$$

where $\tilde{h}(t)=\sum_{p=1}^{P} g_{p} p\left(t-\tau_{p}\right)$. We let

$$
f(t)=\sum_{l=1}^{L} c(l) \tilde{h}\left(t-l T_{c}\right)
$$

$f(t)$ is called the received signature waveform. Then (3) can be written as

$r_{m}(t)=a(i) f(t-i T-\tau) e^{j \phi_{m}}+\sum_{k=1}^{K_{\iota}} \iota_{k}(t) e^{j \psi_{k, m}}+v(t)$.

For the entire duration of transmission, $-N \leq i \leq N$, we have
$r_{m}(t)=\sum_{i=-N}^{N} a(i) f(t-i T-\tau) e^{j \phi_{m}}+\sum_{k=1}^{K_{\iota}} \iota_{k}(t) e^{j \psi_{k, m}}+v(t)$.

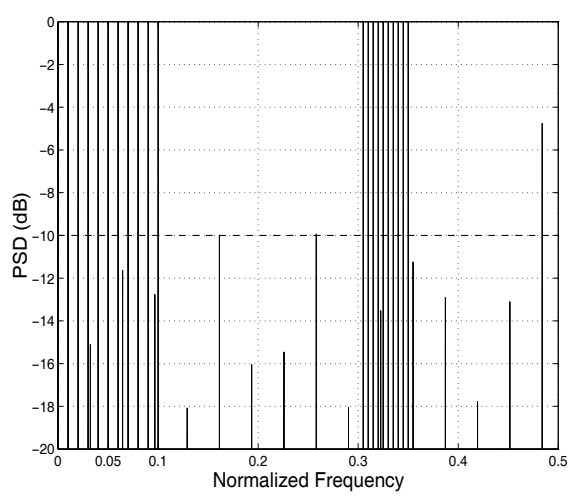

Figure 2: Frequency response of NBI plus SS signal occupying $30 \%$ of $\mathrm{BW}$

\section{SINGLE CHANNEL MMSE ALR STRUCTURE}

For the single antenna case, the ALR consists of a trainingsequence-based adaptive linear MMSE filter and a decision device, Figure 3. Notice that this adaptive filter is structurally similar to the conventional LMS adaptive filter. However, as we stated in the introduction, we process a block of $L$ chip samples to compute one symbol sample at a time. Thus, the output of this filter is given every $L$ chip periods. The adaptation of the filter weights uses a global error for the computation of the weight update. The global error is computed from the difference of the desired signal and the sum of the filters' output.

The receiver has been shown to be robust to multiaccess, narrowband, and multipath interferences. Also it has been shown to be near-far resistant and with complexity independent of the number of users. Moreover, timing, signatures, and carrier phase information from other users is not needed [3]. These generally desirable properties allow this receiver to achieve significant improvement relative to the matched filter receiver. These are the properties that we exploit in this paper.

The output of the filter is given by

$$
y(n T)=\sum_{j=-J}^{J} w(j) r\left(n T-j T_{c}\right)
$$

where $w(j)$ is the $j$-th adaptive filter coefficient and $2 J+1$ is the number of coefficients. The weight adaptation algorithm is the canonical LMS algorithm described by 


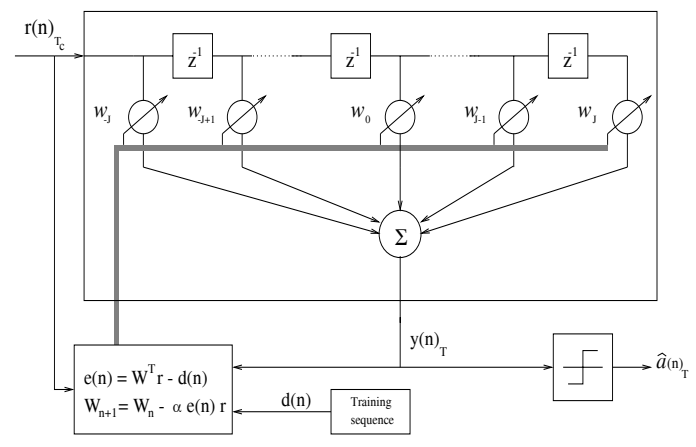

Figure 3: Adaptive Linear Receiver

$$
\mathbf{W}_{n+1}^{(m)}=\mathbf{W}_{n}^{(m)}-\alpha e^{*}(n) \mathbf{X}_{n}^{(m)}
$$

where $\mathbf{W}_{n}^{(m)}$ is the linear filter coefficient sequence for the $m$-th antenna element a the $n$-th iteration, $e(n)$ is the error signal at the $n$-th iteration, and $\alpha$ is the step size.

\section{SIMULATIONS}

In this section we simulate two NBI cases. The first case is a DSSS signal corrupted by a single interference located at $35^{\circ}$ occupying $10 \%$ of the signal bandwidth (BW). The second case is a DSSS signal corrupted by two interferences located at $-45^{\circ}$ and $35^{\circ}$ occupying $30 \%$ of the signal BW. The spatial response of the antenna array is shown in Figure 4 where the thick dotted lines indicate the position of the interferences and the signal of interest. In these simulations we do not include a multipath channel and investigate the performance of the MLAR for AWGN and strong interference only. The received signal is then given by

$$
r_{m}(t)=q(t) e^{j \phi_{m}}+\sum_{k=1}^{K_{\iota}} e^{j \psi_{k, m}}+v(t)
$$

In all the figures below we $\circ$ and $*$ indicate the MALR and the ALR response, respectively. Figure 5 shows the BER performance for the ALR and MALR. The dotted lines represent the BER performance for the AWGN channel case, i.e., no interference. For this simulation we let the interference occupy $10 \%$ of the signal bandwidth with $10 \mathrm{~dB}$ above the signal level. We also let the signal of interest be in the broadside direction of the antenna array. Notice that in both cases the ALR and the MALR achieves comparable performance as the AWGN channel. However, notice that the MALR gains about $7 \mathrm{~dB}$ at a BER of $10^{-4}$ relative to the ALR.

Figure 6 shows the receiver's response to interference power. For this simulation we fix the SNR to $10 \mathrm{~dB}$ and increase the interference power from 0 to $20 \mathrm{~dB}$. The dotted lines represent the response of the single antenna and

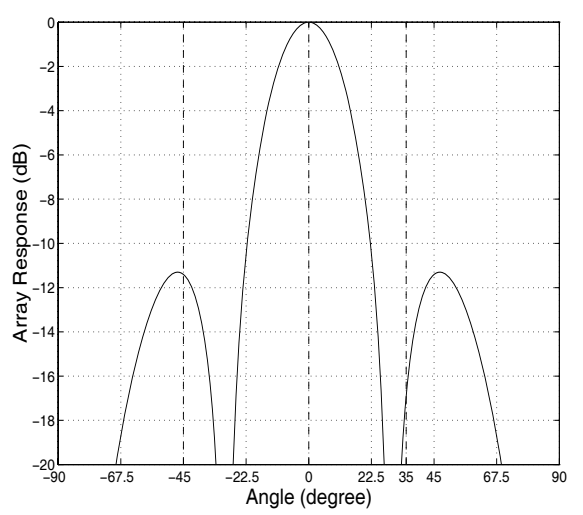

Figure 4: Spatial Array Response

the four antenna receiver without any preprocessing. Notice that below $14 \mathrm{~dB}$ the MALR achieves better BER performance that either the other three cases. However, above 14 $\mathrm{dB}$ the MALR's BER performance degrades rapidly due to convergence problems implying the need of a longer training signal.

Figure 7 shows the BER performance to two independent NBI for the ALR and MALR. The dotted lines represent the BER performance for the AWGN channel case, i.e., not interference. For this simulation we let the interference occupy $30 \%$ of the signal bandwidth with $10 \mathrm{~dB}$ above the signal level. We also let the signal of interest be in the broadside direction of the antenna array. Notice that the MALR gains about $8 \mathrm{~dB}$ at a BER of $10^{-3}$ relative to the ALR.

Finally, Figure 8 shows the BER simulation results for a two NBI case occupying $30 \%$ of the BW as the ISNR is incrased from 0 to $20 \mathrm{~dB}$. We let the interference location be at $35^{\circ}$ and $-45^{\circ}$, relative to the antenna array. The dotted lines represent the BER performance of the single and four antenna without preprocesing. Notice that overall, the MALR has a bertter BER performance.

\section{CONCLUSION}

We showed the feasibility of using an antenna array with MMSE adaptive linear filters for the excision of spatially located NBI. The adaptive filters' processing window spans $3 L$ chip samples to process a symbol sample. Simulation results indicate that the proposed system gains $7 \mathrm{~dB}$ for a single interferer at $10 \mathrm{~dB}$ occupying $10 \%$ of the BW and 8 $\mathrm{dBs}$ for two interferers at $10 \mathrm{~dB}$ each occupying $30 \%$ of the BW. Although for high levels and large frequency occupany, we must consider large training sequences to compensate for slow convergence. 


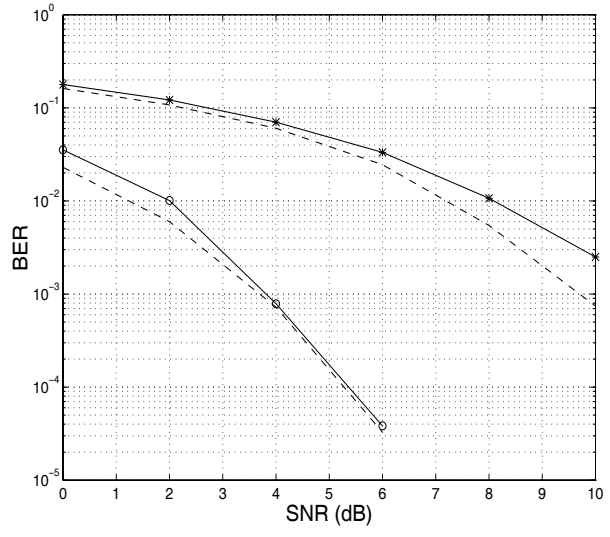

Figure 5: BER for ALR and MALR receiver

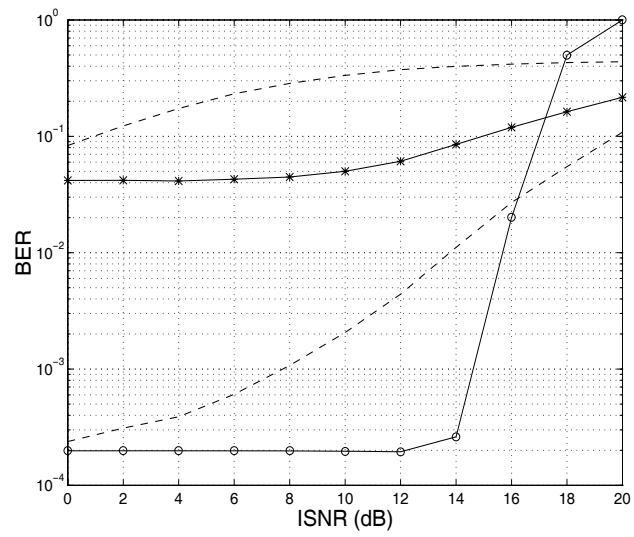

Figure 6: BER vs ISNR for Correlator, ALR and MALR receiver

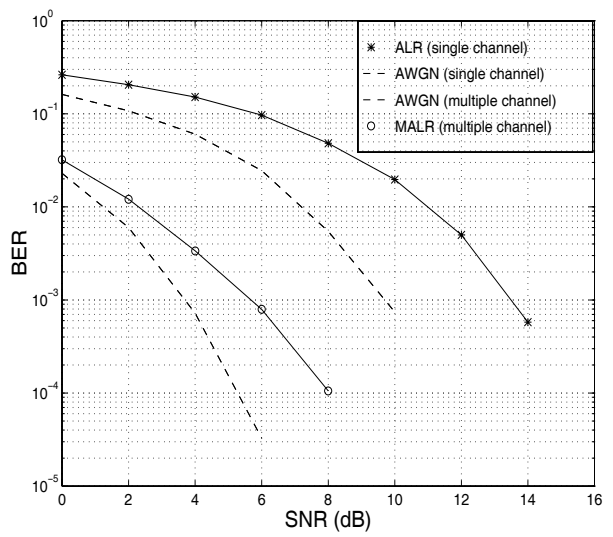

Figure 7: BER for ALR and MALR receiver for 2 NBIs

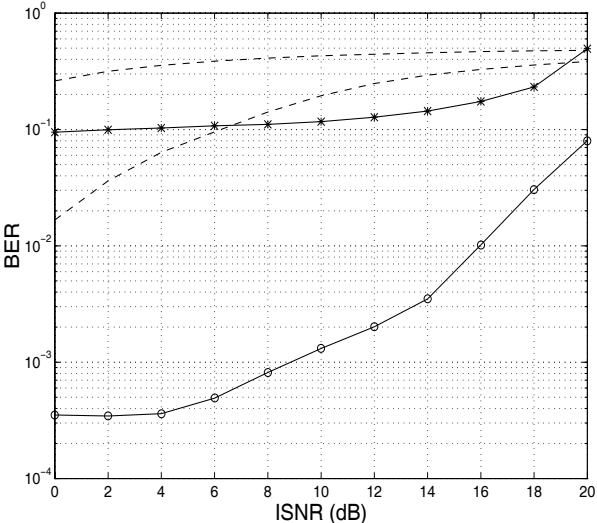

Figure 8: BER vs ISNR for Correlator, ALR and MALR receiver for 2 NBIs

\section{REFERENCES}

[1] J. Winters, "Smart Antennas for Wireless Systems," IEEE Personal Communications, Vol. 5, No. 1, pp. 2327, Feb. 1998.

[2] J. Thompson, P. Grant, and B. Mulgrew, "Smart Antenna Arrays for CDMA Systems," IEEE Personal Communications, Vol. 3, No. 5, pp. 16-25, Oct. 1996.

[3] P. Rapajic and B. Vucetic, "Adaptive Receiver Structures for Asynchronous CDMA Systems," IEEE Journal on Sel. Areas in Comm., Vol. 12, No. 4, pp. 685697, May 1994.

[4] P. Rapajic and B. Vucetic, "Narrow-Band and Multiple Access Interference Rejection by Adaptive Single User Receiver in Asynchronous CDMA Systems," Proceeding of the International Symposium of Information theory and Its Applications (ISITA'94), Sydney,Australia, Vol. 1, pp. 73-78, Nov. 20-25 1994.

[5] Ketchum and Proakis, "Adaptive Algorithms for Estimating and Suppressing Narrow-Band Interference in PN Spread-Spectrum Systems," IEEE Trans. Communications, Vol. COM-30, No. 5, pp. 913-924, May 1982.

[6] Rusch and Poor, "Narrowband Interference Suppression in CDMA Spread Spectrum Communications," IEEE Trans. Communications, Vol. COM-42, No. 2/3/4, pp. 1969-1979, Feb/March/April 1994. 